\title{
Sensitivity and packaging improvement of an LCP pressure sensor for intracranial pressure measurement via FEM simulation
}

\author{
Preedipat Sattayasoonthorn $^{1}$, Jackrit Suthakorn ${ }^{2}$, Sorayouth Chamnanvej ${ }^{3}$ \\ ${ }^{1}$ Department of Biomedical Engineering, Faculty of Engineering, Mahidol University, Thailand \\ ${ }^{2}$ Center for Biomedical and Robotics Technology, Faculty of Engineering, Mahidol University, Thailand \\ ${ }^{3}$ Department of Surgery, Faculty of Medicine Ramathibodi Hospital, Mahidol University, Thailand
}

\section{Article Info}

Article history:

Received Feb 26, 2019

Revised Apr 24, 2019

Accepted Apr 30, 2019

\section{Keywords:}

Finite element method Intracranial pressure sensor LCP MEMS fabrication Simulation

\begin{abstract}
A biocompatible liquid crystal polymer (LCP) pressure sensor is proposed for measuring intracranial pressure (ICP) in Traumatic Brain Injury (TBI) patients. Finite element method using COMSOL multiphysics is employed to study the mechanical behavior of the packaged LCP pressure sensor in order to optimize the sensor design. A $3 \mathrm{D}$ model of the $8 \times 8 \times 0.2 \mathrm{~mm} \mathrm{LCP}$ pressure sensor is simulated to investigate the parameters that significantly influence the sensor characteristics under the uniform pressure range of 0 to $50 \mathrm{mmHg}$. The simulation results of the new design are compared to the experimental results from a previous design. The result shows that reducing the thickness of the sensing membrane can increase the sensitivity up to six times of that previously reported. An improvement of fabrication methodology is proposed to complete the LCP packaging.
\end{abstract}

Copyright $@ 2019$ Institute of Advanced Engineering and Science. All rights reserved.

\section{Corresponding Author:}

Jackrit Suthakorn,

Center for Biomedical and Robotics Technology, Faculty of Engineering,

Mahidol University, 25/25 Puttamonthon 4 Road,

Salaya, Nakorn Pathom 73170, Thailand.

Email: jackrit.sut@mahidol.ac.th

\section{INTRODUCTION}

Patients with severe traumatic brain injury are required to have their intracranial pressure (ICP) monitored. As the brain swells the intracranial pressure rises, an ICP of over $15 \mathrm{mmHg}$ can harm the brain and the spinal cord $[1,2]$. Several conventional systems are used to measure ICP, with each system being suitable for a particular case. Different devices are placed in different areas, the location being dependent on the patient's condition. Existing devices have limitations, such as: risk of infection, high cost and risk of bleeding [3-5].

New ICP monitoring devices aim to reduce the risk of infection [6]. Minimally invasive pressure sensors should be miniature in size, transmit data wirelessly and be low cost. In previous work a Liquid Crystal Polymer or LCP pressure sensor was proposed [7]. LCP is an appealing material for this application as it is biocompatible, has low moisture absorption and it has a wide range of chemical resistances. Reported applications of LCP in a biological environment include: the use of LCP as a flexible electrode for neural stimulation [8-10], as an interconnect for an implanted device [11], and as packaging for an implantable sensor [12]. Given that medical grade LCP sheet is easily available [13], it is an attractive material for the minimally invasive measurement of ICP.

In a previous study [7, 14], an LCP pressure sensor was designed using the square plate theory under small deformation condition. Given that LCP shows a high degree of isotropy, the material was assumed to display linearly elastic behavior, based on this assumption the membrane deflection under 
uniform applied pressure was evaluated to optimize the sensing membrane size. A Comsol-based finite element analysis was used to investigate the membrane deflection. The model only considered the top of the LCP membrane with the dimensions shown in Figure 1. The simulation was performed with a boundary load condition. The membrane deflection was simulated using pressures ranging from 0 to $50 \mathrm{mmHg}$. The results showed that the LCP membrane deformed elastically and did not break under maximum pressure. The maximum deflection of the LCP membrane did not exceed the outer membrane's thickness which shows that the sensor can operate in higher pressures as well. The results also showed that the maximum deflection occurred at the center of the membrane and the maximum stress (strain) occurred at the center of the membrane edges. The strain gauges were therefore designed to be placed on these edges to capture this high strain.

The gold strain gauges were designed as a built in Wheatstone bridge. A serpentine shape was used to increase the resistance of the total strain gauge. A prototype LCP pressure sensor was fabricated and tested in a hydrostatic environment. The measured resistance was approximately $60 \%$ more than the designed nominal value, however, the study identified a drawback to the design which led to low sensitivity and a limit to the operating pressure range. The sensitivity of the previous design was not sufficient, and the packaging was not fully water tight. The present study offers two approaches to overcome the aforementioned shortcomings of the previous work: the first approach aims to improve the performance of the sensor to increase the sensitivity. In this case, the finite element method is chosen to study the mechanical behaviors of the LCP membrane deformation under the operating pressure range. This study suggests the appropriate sensor design which generates higher sensitivity than the previous one, details are given in Section 2.

The second approach aims to improve the sensor packaging to avoid water leakage into the sensor's cavity. A new fabrication process is proposed to achieve a complete LCP ICP monitoring package as shown in Figure 1. The microfabrication process is primarily used to develop the polymer-based fabrication process $[15,16]$. The contributions of this work are as follows: It is shown through finite element analysis that it is possible to achieve a sensitivity of $0.314 \mathrm{mV} / \mathrm{mmHg}$ using a sensor membrane thickness of $20 \mu \mathrm{m}$ for a pressure range of 0 to $50 \mathrm{mmHg}$ with the sensor membrane being fabricated from LCP (Section 3). An improved recipe for LCP pressure sensor fabrication is presented (Section 4). A complete miniature, biocompatible sensor package is proposed (Section 5). Therefore, the novel technical results presented in this work are twofold: firstly, an improved design of an LCP pressure sensor is given which leads to an increase in the sensor's sensitivity of up to six times that of the previous design. Secondly, an improved recipe of the LCP pressure sensor's fabrication is presented that is expected to yield a complete and water-tight packaging solution.

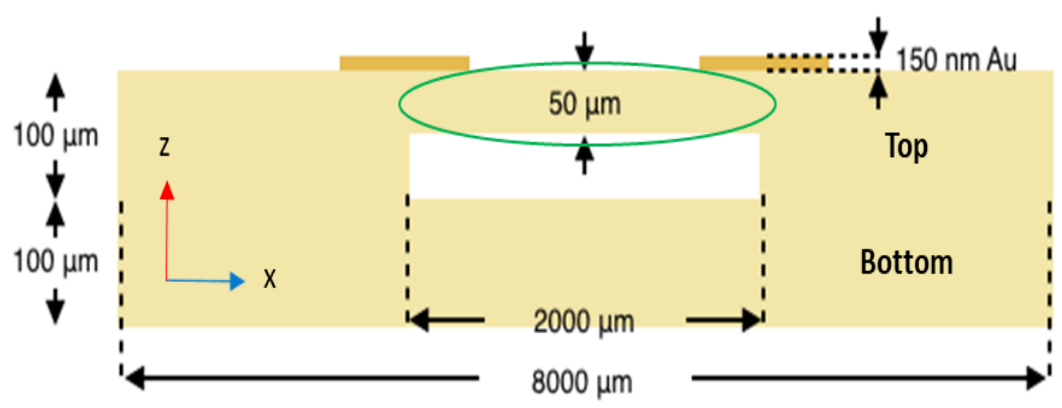

Figure 1. Cross-sectional drawing of the LCP pressure sensor consisting of top membrane with sensing membrane (in green ellipse) and a sealed pressure chamber

\section{RESEARCH METHOD}

According to the previous work [14], the top LCP membrane consists of the $50 \mu \mathrm{m}$ sensing membrane and bottom cavity which is required to be sealed to prevent the fluid flow into the air cavity. The LCP pressure sensor is designed to use LCP packaging due to the advantage of biocompatibility so a 100 $\mu \mathrm{m}$ thick LCP membrane is used to seal the air cavity by heat bonding. The bonding technique is described in the next section. Since the applied pressure occurs on the top membrane, the effects on membrane deflection and stress are studied to investigate their distribution. The conclusions of the previous study suggested that the sensitivity of the device can be improved by decreasing the thickness of sensing membrane, the variation of sensing membrane thickness is therefore varied to determine the appropriate thickness to provide a higher sensitivity. 
Modeling and simulation are useful for micro [17] and nano-scale electronic design [18]. A 3D model of the LCP pressure sensor is built using COMSOL Multiphysics (v4.3). The total width and thickness of the top and bottom membranes are $8 \mathrm{~mm}$ and $0.2 \mathrm{~mm}$ respectively. For the top membrane, the chamber is sealed with $100 \mu \mathrm{m}$ thick LCP bottom membrane. The sensing membrane thickness is varied from 15 to $50 \mu \mathrm{m}$ in steps of $5 \mu \mathrm{m}$ to find the optimal deflection that does not exceed the outer membrane thickness. The structural mechanics module is applied to the 3D model in stationary analysis. LCP is assumed a linear elastic material, all parts are analyzed as linear elastic based on the small deformation assumption. The solid mechanics interface is used to quantify and characterize the stress and displacement. The material properties are set with ULTRALAM 3850 LCP sheet from Roger Corporation [8] and listed in Table 1. A fixed constraint is applied to the bottom of the device to avoid the displacement of the overall part.

Table 1. Material Properties and Device's Dimension used in FEM

\begin{tabular}{ll}
\hline Properties & Value \\
\hline Material & Liquid Crystal Polymer (LCP) \\
Density $(\square)$ & $1.4 \mathrm{gm} / \mathrm{cm}^{3}$ \\
Young's Modulus (E) & $2255 \mathrm{MPa}$ \\
Poisson's ratio ( $\square$ ) & 0.3 \\
Device dimension & $8 \times 8 \times 0.2 \mathrm{~mm}$ \\
Sensing membrane dimension & $2 \times 2 \times \mathrm{T} * \mathrm{~mm}$ \\
$* \mathrm{~T}$ is the variable thickness in the range of $20-50 \mu \mathrm{m}$ & \\
\hline
\end{tabular}

A boundary load is applied to model the external pressure changes around the device. This external pressure corresponds to the change of the volume of the cavity which results in the pressure changes inside the air cavity. Hence, the internal pressure change as a function of the volume is defined in another boundary condition applied in the air cavity. The internal pressure change $(\Delta P)$ is written in (1) [19]:

$$
\triangle \mathrm{P}=P_{0}\left(\left(\frac{V_{0}}{V}\right)^{\gamma}\right)-1
$$

where $P_{0}$ is the ambient pressure in air cavity, $V_{0}$ is the undeformed volume, $V$ is the deformed volume under the external pressure change and $\gamma$ is the adiabatic index of air. In this case, $P_{0}=1 \mathrm{~atm}, V_{0}=2 \times 10^{-6} \mu \mathrm{m}^{3}$ and $\gamma=1.4$. The outputs of the simulation are the sensing membrane displacement, stress and strain under a pressure of $50 \mathrm{mmHg}$. The sensing membrane displacement is evaluated to find its optimal thickness, where the sensing membrane is at the center of the top membrane as shown in Figure 1. The maximum stress is simulated to evaluate the acceptable thickness that does not break the membrane. The strain is evaluated to identify the appropriate region on the membrane for placing the strain gauges. After the optimization the strain variation is evaluated with respect to a pressure of 0 to $50 \mathrm{mmHg}$ to characterize the effect of varying the membrane thickness.

The purpose of this present work is to improve the sensitivity of the previous design, the maximum strain and output voltage are used to observe the sensor response and compare the efficiency between the previous and present designs. The results of simulated strain are used to calculate the relative changes in resistance for the resistors in order to obtain the sensor sensitivity. The change in strain from the FEM analysis is used to calculate the sensor's output voltage when varying the sensing membrane's thickness.

$$
\frac{\mathrm{V}_{\mathrm{o}}}{\mathrm{V}_{\mathrm{s}}} \approx \frac{-\alpha_{4}-\alpha_{1}-\alpha_{4} \alpha_{1}}{4}
$$

where $V_{o}$ and $V_{s}$ are the output voltage and supply voltage respectively. $\alpha_{1}$ and $\alpha_{4}$ are the relative changes in resistance for the resistors in terms of the surface strain and can be found from the relative change in resistance for a resistor segment deformed by being bonded to the top of the plate [14].

\section{RESULTS AND ANALYSIS}

The FEM results show the deflection, stress and strain of the membrane under the pressure range of 0 to $50 \mathrm{mmHg}$. The results of the simulated strain are used to determine the sensitivity of the sensor which is compared to the experimental results from the previous design. 


\subsection{Finite element method for mechanical properties analysis}

Figures 2, 3 and 4 show the simulations of membrane deflection, stress and strain as a function of membrane thickness under a pressure of $50 \mathrm{mmHg}$ respectively. In Figure 2, if the membrane is thinner than $20 \mu \mathrm{m}$, the membrane will deflect such that it reaches the bottom membrane, therefore for this sensor design the sensing membrane can only be considered in the range of 20 to $50 \mu \mathrm{m}$. The tensile strength of LCP membrane is $282 \mathrm{MPa}$, Figure 3 shows the simulation of maximum stress in the x-direction, it can be seen that the stress does not exceed this value for the membrane thicknesses of 20 to $50 \mu \mathrm{m}$, as such the membrane is not expected to fail. Figure 4 shows that the maximum strain is found at the edges of the membrane.

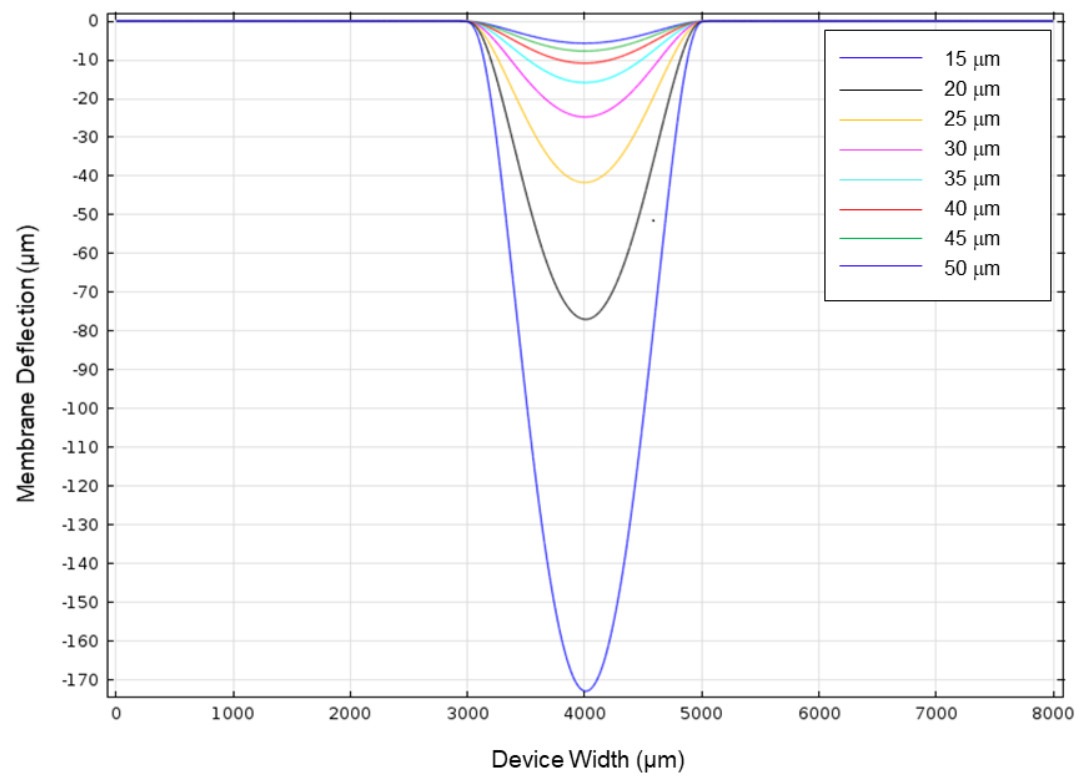

Figure 2. LCP membrane deflection for different thickness with a pressure of $50 \mathrm{mmHg}$ in the cross-section along the width

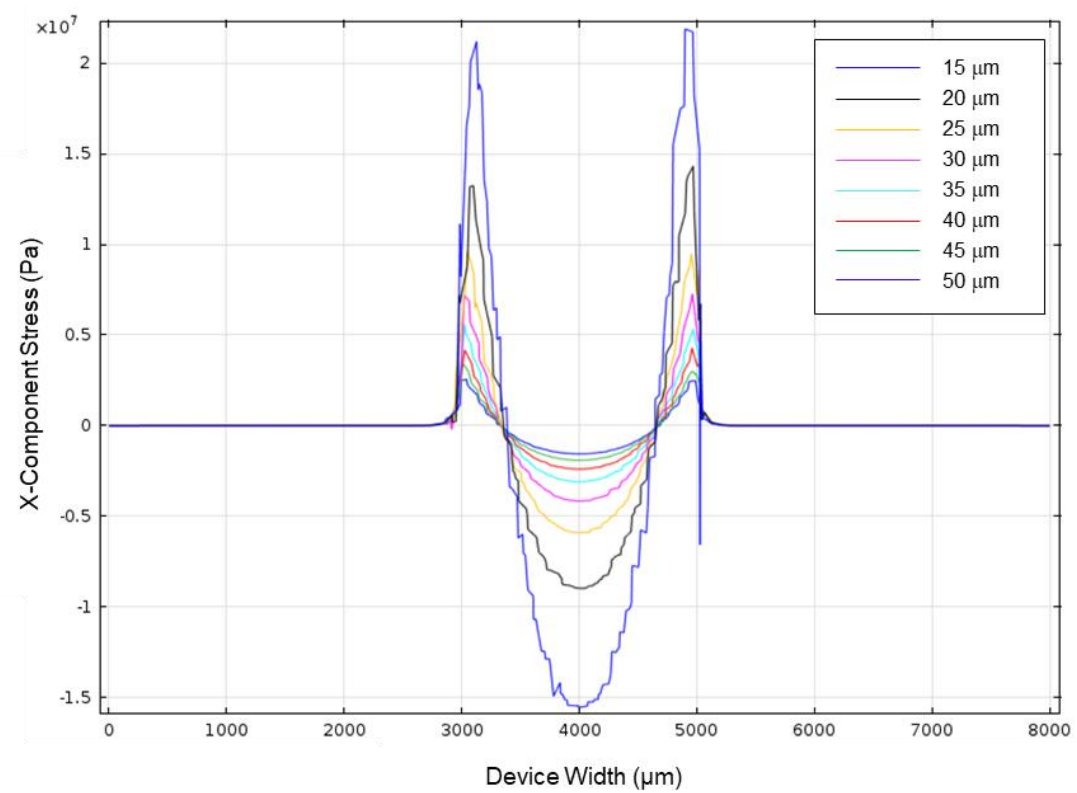

Figure 3. X-component of the LCP membrane stress as the function of membrane thickness under a pressure of $50 \mathrm{mmHg}$ in the cross-section along the width 


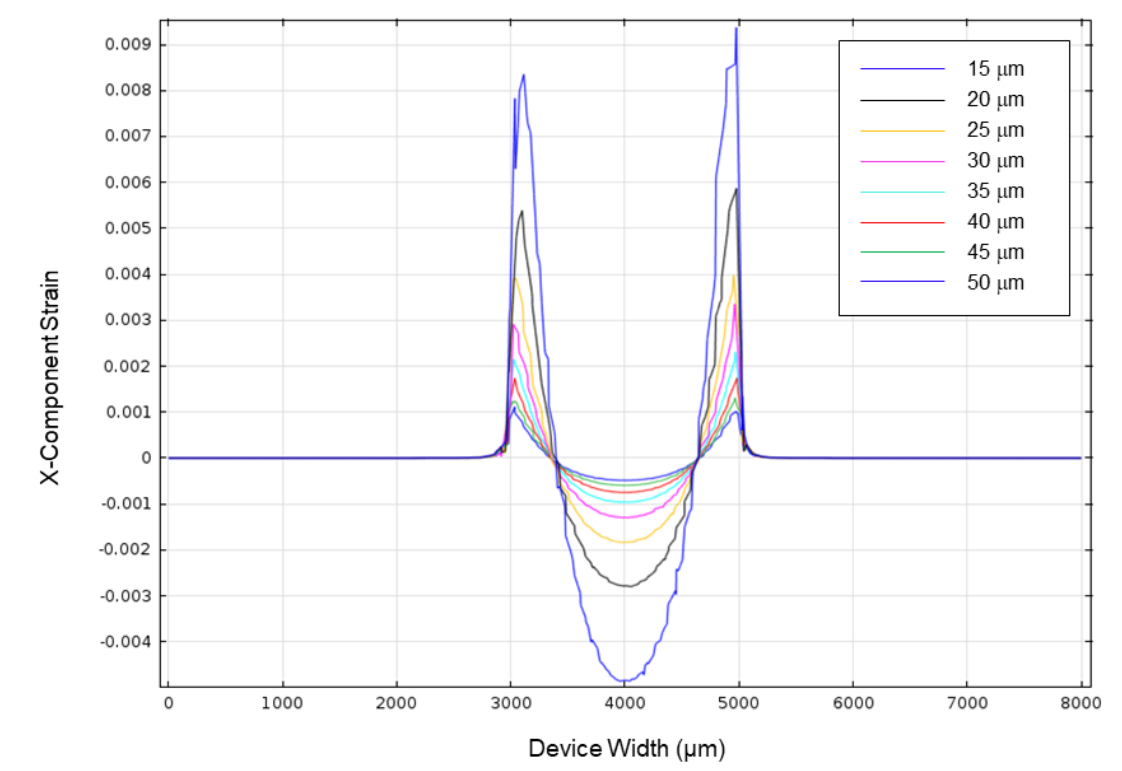

Figure 4. X-component of the LCP membrane strain under a pressure of $50 \mathrm{mmHg}$ in the cross-section along the width

Having selected the acceptable range of sensing membrane thicknesses, Figure 5 shows the simulated results of the strain variation as function of pressure for different sensing membrane thicknesses. The pressure is varied from 0 to $50 \mathrm{mmHg}$. The strain evolves from $0.02 \%$ at $10 \mathrm{mmHg}$ to $0.12 \%$ at $50 \mathrm{mmHg}$ for $50 \mu \mathrm{m}$. In the case of $20 \mu \mathrm{m}$, the strain varies from $0.15 \%$ at $10 \mathrm{mmHg}$ to $0.73 \%$ at $50 \mathrm{mmHg}$. Figure 6 shows the evolution of strain versus the thickness of sensing membrane varying from 20 to $50 \mu \mathrm{m}$ under a pressure of $50 \mathrm{mmHg}$.

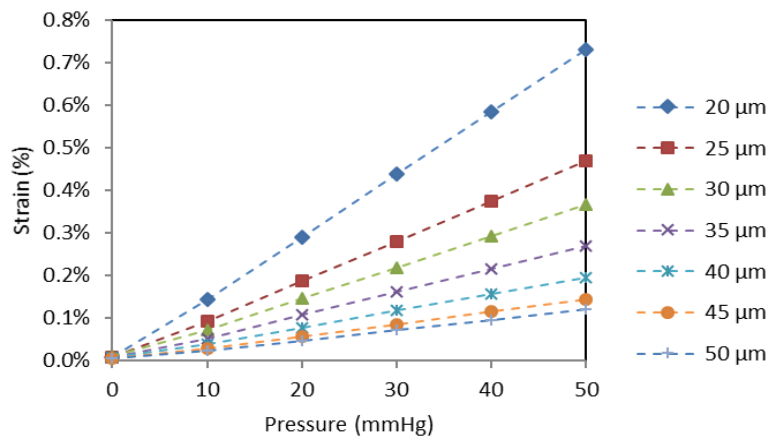

Figure 5. The simulated strain variation versus pressure for various sensing membrane thicknesses

\subsection{Sensor response analysis}

The output voltage is calculated by using the result of simulated strain from the FEM. The results of output voltage are plotted as a function of pressure for different sensing membrane thickness as shown in Figure 7. At $50 \mathrm{mmHg}$ the evolution of output voltage is plotted as a function of sensing membrane thickness, see Figure 8. The sensitivity can be obtained from the gradient of the output voltage versus pressure graph. The sensitivity presents the optimized design to quantify the measurement efficiency of the pressure sensor. From the graphs it can be seen the best sensitivity occurs with the thinnest sensing membrane of $20 \mu \mathrm{m}$ which corresponds to the highest strain in Figure 6.

To evaluate the improvement, the sensitivity of simulated results and experimental result are used to plot the output voltage at 0 to $30 \mathrm{mmHg}$ pressure in Figure 9. The simulated results are presented at 20 and $50 \mu \mathrm{m}$ thick sensing membrane which are 0.314 and $0.049 \mathrm{mV} / \mathrm{mmHg}$ respectively. The experimental result is obtained from the previous design with a $50 \mu \mathrm{m}$ thick sensing membrane and shown as $0.048 \mathrm{mV} / \mathrm{mmHg}$. 
The evolution of the output voltages is summarized at the pressure of 5 and $30 \mathrm{mmHg}$ in Table 2 . The span of variation implies the higher sensitivity of the present design. Hence, the $20 \mu \mathrm{m}$ thick sensing membrane is selected for the sensing membrane fabrication.

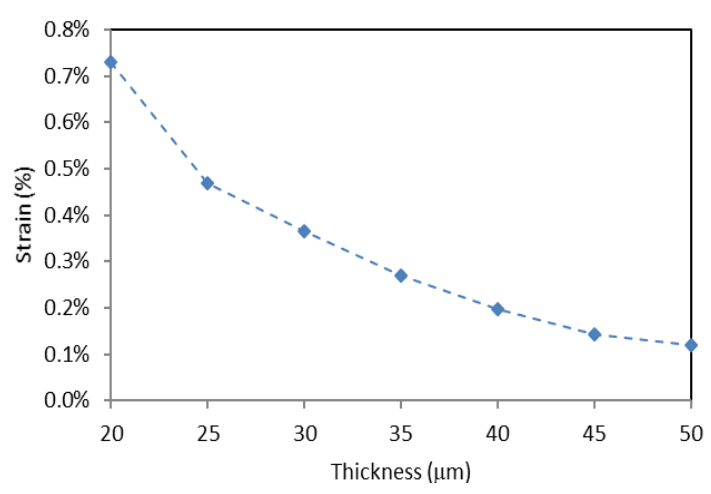

Figure 6. Evolution of simulated strain for various sensing membrane thickness under a pressure of $50 \mathrm{mHg}$

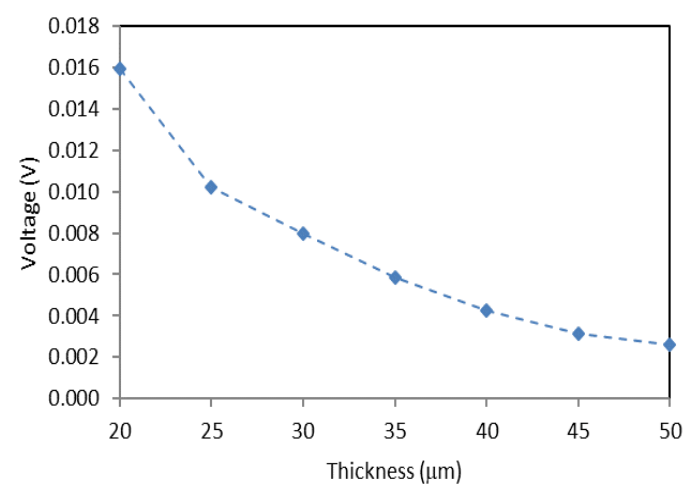

Figure 8. Evolution of calculated output voltage for various sensing membrane thickness under a pressure of $50 \mathrm{mmHg}$

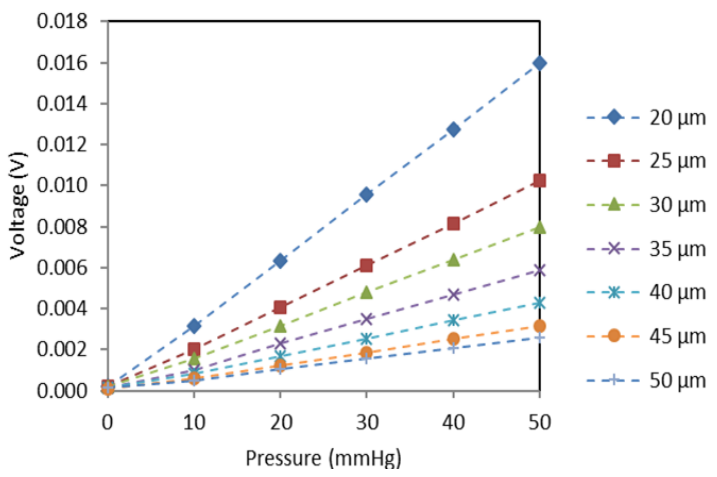

Figure 7. The calculated output voltage variation versus pressure for various sensing membrane thicknesses

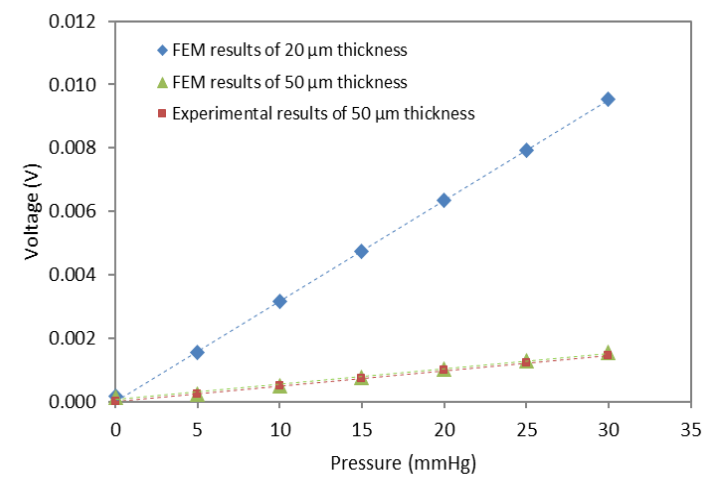

Figure 9. Comparative sensitivity for present and previous designs under different pressures

Table 2. The experimental and simulated results of the output voltages at 20 and $50 \mu \mathrm{m}$ thickness

\begin{tabular}{cccc}
\hline $\begin{array}{c}\text { Pressure } \\
(\mathrm{mmHg})\end{array}$ & $\begin{array}{c}\text { Experimental output voltage } \\
\text { at } 50 \mu \mathrm{m} \text { thickness }(\mathrm{mV})\end{array}$ & $\begin{array}{c}\text { Simulated output voltage } \\
\text { at } 20 \mu \mathrm{m} \text { thickness }(\mathrm{mV})\end{array}$ & $\begin{array}{c}\text { Simulated output voltage } \\
\text { at } 50 \mu \mathrm{m} \text { thickness }(\mathrm{mV})\end{array}$ \\
\hline 5 & 0.244 & 1.559 & 0.251 \\
30 & 1.463 & 9.536 & 1.558 \\
\hline
\end{tabular}

\section{PROPOSED PACKAGING}

The device is designed to be fabricated entirely from LCP, ULTRALAM 3850 LCP sheet from Roger Corporation [20] is used to fabricate the core layer. Previous work has proved the validity of the fabrication processes and the recipes with LCP, however, improper packaging was discussed as the cause of potential water leakage into the cavity of the pressure sensor. In this case, the present design is proposed to improve upon the previous LCP package fabrication. LCP bondply is applicable to use in multilayer construction and lamination [21] since the electrical properties and moisture absorption are similar to ULTRALAM 3850. The low and stable dielectric constant and signal loss make the bondply suitable for high frequency applications in telecommunication. The low elastic modulus property offers the benefit in mechanical flexibility. The melting temperature of LCP bondply is approximately $285^{\circ} \mathrm{c}$ which is less than that of ULTRALAM $3850\left(315^{\circ} \mathrm{c}\right)$, so this property facilitates the process of thermal bonding. ULTRALAM 3908 bondply sheet is used to adhere the core layers. 
The present design consists of two core layers (top and bottom) and an adhesive layer. The top core layer is fabricated as shown in Figure 10. A $100 \mu \mathrm{m}$ thick LCP copper clad is temporarily attached to a silicon wafer by using photoresist and transferred the square mask onto the top surface as shown in Figure 10(a). The bare LCP between the square mask is etched to build the $20 \mu \mathrm{m}$ thick sensing membrane and the cavity. The top copper layer is then etched off as shown in Figure 10(b). The top core layer is removed from the silicon wafer by using acetone as shown in Figure 10(c).

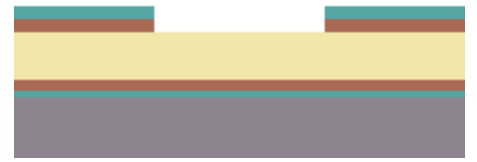

(a)

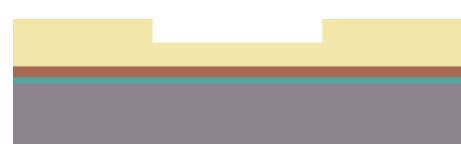

(b)

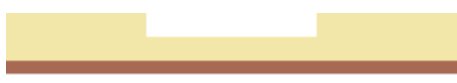

(c)

Figure 10. Top core layer fabrication: (a) Etched and square-patterned LCP sheet for LCP etching process, (b) $20 \mu \mathrm{m}$ thick sensing membrane after LCP etching, and (c) Top core layer after removing photoresist

The bottom core layer is fabricated as shown in Figure 11. Another $100 \mu \mathrm{m}$ thick LCP copper clad is temporarily attached to a silicon wafer to etch the top copper layer as shown in Figure 11(a). The bottom core layer is removed from the support wafer as shown in Figure 11(b). Next, ULTRALAM 3908 bond ply is cut to make $2 \times 2 \mathrm{~mm}^{2}$ window and alignment marks with a $\mathrm{CO}_{2}$ laser.

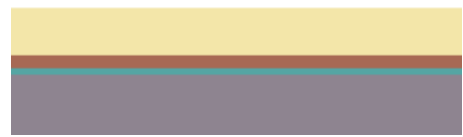

(a)

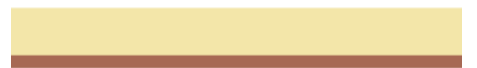

(b)

Figure 11. Bottom core layer fabrication: (a) Etched top copper layer of LCP sheet and (b) Bottom core layer after removing photoresist

The bondply sheet is sandwiched between the core layers by using alignment marks and passed through the thermal bonding as shown in Figure 12(a). Both copper layers are etched off as shown in Figure 12(b). The top core layer is deposited with the photoresist and transferred the serpentine mask by using backside alignment in photolithography process. Ti/Au is sputtered on the surface to make metallic strain gauges as shown in Figure 12(c) and passed to the liftoff process to remove photoresist and leave the strain gauges as shown in Figure 12(d).

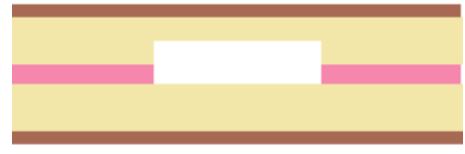

(a)

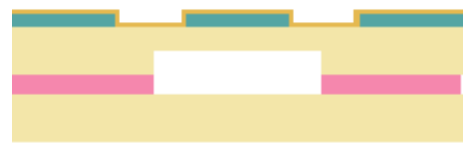

(c)

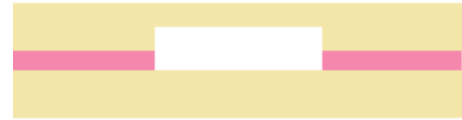

(b)

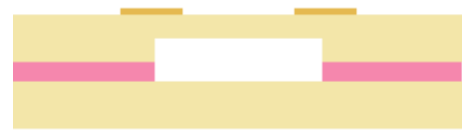

(d)

Figure 12. Packaging for LCP pressure sensor: (a) Sandwiched construction of top core layer, bondply and bottom core layer after thermal bonding, (b) Exposed LCP after copper etching, (c) Photoresist deposition and Ti/Au sputtering on the top core layer, and (d) Bonded LCP pressure sensor with gold serpentine strain gauges after lift-off process 


\section{DISCUSSION}

A packaged LCP pressure sensor is proposed and analyzed for ICP monitoring. This present study shows that decreasing the thickness of the LCP sensing membrane can improve the sensitivity of the device. The simulation results at $20 \mu \mathrm{m}$ thick sensing membrane indicates more than a six-fold increase in sensitivity when compared to experimental results with a $50 \mu \mathrm{m}$ thick sensing membrane that was reported in previous work [14]. There are other alternative ways to possibly improve the sensitivity such as increasing the number of turns of the serpentine strain gauge, which can be done by decreasing the strain gauge width. Another way to increase sensitivity is changing the placement of strain gauges to be on the sensing membrane. The proposed fabrication process will lead to a complete LCP structure which can be used as a miniature and biocompatible sensor. A hermetic package will be considered in further fabrication process. In future work, the completed LCP sensing unit will be integrated with the data transmission unit that includes the power source of the sensor. LCP sheet will be used to support the electrical components and encapsulate the data transmission unit. Although it is well known that reducing the sensor membrane thickness improves the sensitivity of a sensor, it has been shown in this work that using a $20 \mu \mathrm{m}$ LCP pressure sensor thickness will yield a fully functional sensor over the required pressure range for ICP monitoring. This result has not been presented elsewhere.

\section{CONCLUSION}

This work presents a detailed design, simulation-based characterization, and proposed fabrication process of a packaged LCP pressure sensor for intracranial pressure sensing. The design is studied and optimized to improve the sensitivity of the sensor. The major physical characteristics and device behavior are modeled via FEM. The study shows that the proposed design of a $20 \mu \mathrm{m}$ thick sensing membrane can operate under the pressure range of 0 to $50 \mathrm{mmHg}$ with a sensitivity of $0.314 \mathrm{mV} / \mathrm{mmHg}$. A detailed fabrication process is proposed to complete the LCP package. The proposed design represents a step towards realizing a miniature biocompatible wireless pressure sensor for the use in ICP monitoring and healthcare applications.

\section{ACKNOWLEDGEMENTS}

This research has been funded by FY2016 Thesis Grant for Doctoral Degree Student under National Research Council of Thailand (NRCT) through Mahidol University.

\section{REFERENCES}

[1] "Surgeons BTFAA of NSC of N. Guidelines for the management of severe traumatic brain injury 3rd edition," Journal of neurosurgery, vol/issue: 24(212), pp. S1-106, 2007. Available: http://www.ncbi.nlm.nih.gov/pubmed /17511534.

[2] Steiner L. A. and Andrews P. J. D., "Monitoring the injured brain: ICP and CBF," British Journal of Anaesthesia, vol/issue: 97(1), pp. 26-38, 2006

[3] "Recommendations for Intracranial Pressure Monitoring Technology," Journal of Neurotrauma, vol/issue: 17(6-7), pp. 497-506, 2000. Available from: http://www.liebertonline.com/doi/abs/10.1089/neu.2000.17.497.

[4] Lavinio A. and Menon D. K., "Intracranial pressure: Why we monitor it, how to monitor it, what to do with the number and what's the future?" Current Opinion in Anaesthesiology, vol/issue: 24(2), pp. 117-23, 2011.

[5] Zhong J., et al., "Advances in ICP monitoring techniques," Neurological Research, vol/issue: 25(4), pp. 339-50, 2003. Available from: http://www.tandfonline.com/doi/full/10.1179/01616410 3101201661.

[6] Kawoos U., et al., "Too much pressure: wireless intracranial pressure monitoring and its application in traumatic brain injuries," Scientific American, vol/issue: 16(2), pp. 39-53, 2015.

[7] Sattayasoonthorn P., et al., "LCP MEMS implantable pressure sensor for Intracranial Pressure measurement," in The 7th IEEE International Conference on Nano/Molecular Medicine and Engineering, IEEE, pp. 63-7, 2013. Available from: http://ieeexplore.ieee.org/document/6766317/.

[8] Min K. S., et al., "A liquid crystal polymer-based neuromodulation system: An application on animal model of neuropathic pain," Neuromodulation, vol/issue: 17(2), pp. 160-9, 2014.

[9] Wang K., et al., "Flexible nerve stimulation electrode with iridium oxide sputtered on liquid crystal polymer," IEEE Transactions on Biomedical Engineering, vol/issue: 56(1), pp. 6-14, 2009.

[10] Lee S. E., et al., "A flexible depth probe using liquid crystal polymer," IEEE Transactions on Biomedical Engineering, vol/issue: 59(7), pp. 2085-94, 2012.

[11] R. N. Dean, et al., "Novel biomedical implant interconnects utilizing micromachined LCP," in Proceedings of SPIE, pp. 88-99, 2004. Available from: http://link.aip.org/link/?PSI/5515/88/1\&Agg=doi\%5Cnhttp://proceedings. spiedigitallibrary.org/proceeding.aspx?doi=10.1117/12.559831

[12] Qin Y., et al., "Polymer integration for packaging of implantable sensors," Sensors and Actuators, B: Chemical, vol. 202, pp. 758-78, 2014. Available from: http://dx.doi.org/10.1016/j.snb.2014.05.063.

[13] Wang X., et al., "Liquid crystal polymer for MEMS: Processes and applications," Journal of Micromechanics and 
Microengineering, vol. 13, pp. 628-633, 2003. Available from: http://www.iop.org/EJ/abstract/0960-1317/13/5/314

[14] Sattayasoonthorn P., et al., "On the Feasibility of a Liquid Crystal Polymer Pressure Sensor for Intracranial Pressure Measurement,” Biomedical Engineering/Biomedizinische Technik. (in press).

[15] Velten T., et al., "Packaging of bio-MEMS : Strategies, technologies, and applications," IEEE Transactions on Advanced Packaging, vol/issue: 28(4), pp. 533-46, 2005.

[16] Mapato M., et al., "Metal-embedded SU-8 slab techniques for low-resistance micromachined inductors," International Journal of Electrical and Computer Engineering, vol/issue: 8(6), pp. 4772-80, 2018.

[17] Cabello-ruiz R., et al., "Displacement mechanical amplifiers designed on poly-silicon," International Journal of Electrical and Computer Engineering (IJECE), vol/issue: 9(2), pp. 894-901, 2019.

[18] Bouneb I. and Kerrour F., "Nanometric modelization of gas structure, multidimensional using comsol software," International Journal of Electrical and Computer Engineering, vol/issue: 8(4), pp. 2014-20, 2018.

[19] Ohanian H. C. and Markert J. T., "Physics for Engineers and Scientists (Third Edition)," pp. 647-9, 2007. Available from: http://www.amazon.com/dp/0393929698.

[20] Rogers Corporation, "ULTRALAM® 3000 flexible copper clad laminate and bondply," 2016. Available from: https://www.rogerscorp.com/documents/730/acm/ULTRALAM-3000-LCP-laminate-data-sheet-ULTRALAM3850.aspx

[21] Kingsley N., "Liquid crystal polymer : Enabling next-generation conformal and multilayer electronics," Microwave Journal, vol. 51, pp. 188-200, May 2008. 